\title{
The State of Litter of Pigs in the Conditions of the Complex
}

\author{
Romazan Siraziev ${ }^{1}$ and Olesia Gruzdova ${ }^{1, *}$ \\ ${ }^{1}$ Irkutsk Interregional Veterinary Laboratory, Russia \\ ${ }^{2}$ Far Eastern State Agrarian University ,86, Politeknicheskaya Str., Blagoveschensk, Russia
}

\begin{abstract}
The state of litter of large white pigs in industrial conditions was studied. Embryonic mortality is higher during the first 30 days of littering $(21.6 \%)$ - in the period of histotrophic feeding of fetuses - before the beginning of placental connection formation. whereas during the whole period of pregnancy it is $38.4 \%$. In a number of sows at different periods of littering. the number of corpus luteum and the number of fetuses from the side of the corresponding fertile horn do not coincide. that which indicates the migration of embryos into contralateral horn in the early timing of pregnancy. Fetuses growth rate is higher by the 45-days pregnancy. In the litter of pigs there are normotrophic (full-fledged. largefetused). hypotrophic (small-weight) and stillborn pigs. There is close inverse relationship $(r=-0.98$ between fertility and full-fledgeness of piglets в гнездеin the nest. wherein the number of hypotrophic and stillborn ones as the number of newborns in the litter increases has direct positive correlation $(\mathrm{r}=+0.98)$. The more piglets in the litter. the fewer full-fledged ones. and on the contrary. the number of hypotrophic and stillborns is increasing.
\end{abstract}

\section{Introduction}

In the practice of breeding pigs. different breeding traits are used. but the most difficult is the selection for reproductive qualities. It has been established that in domestic swine massifs. multiple births are 10-12 piglets. imported breeds (Landrace and Yorkshires of Danish origin) have 13-14 piglets at farrowing. and in 35-40\% of cases they can give birth to 16-18 or more per farrowing. [14]. According to N.A. Loban and others [4]. large white pigs in Belarus. when surveyed. have an average of 10.7 piglets per farrowing.

The majority of domestic producers have already purchased overseas replacement young animals with improved reproductive qualities. This means that the average multiple sows have to be at least 12 live piglets per farrow. The natural physiology of sows cannot be changed and therefore it is easy to calculate how many piglets a sow can produce. The time from insemination to farrowing averages 114 days. the suckling period lasts 30 days. and after weaning the piglets. a healthy sow comes into heat on the 3rd-8th day. Thus. the

\footnotetext{
${ }^{*}$ Corresponding author: *gruzdova76@mail.ru
} 
complete cycle is at least 150 days. The sow will have 2.43 such cycles per year. Taking into account the sows that. for some reason. could not farrow or remained uncrowded. the average farrowing index will be 2.3 times a year. or 27.6 piglets per average annual sow. With an average level of losses of the received young stock of $12 \%$. at least 24 pigs fed up to $100-110 \mathrm{~kg}$ will reach the slaughterhouse from this sow. which is almost 2.5 tons. But not everything is so simple in domestic pig breeding [1. 12].

The reproductive qualities of pigs are assessed according to 20 or more indicators: fertility. duration of pregnancy. number of nipples in a sow. embryonic mortality. potential and actual multiple pregnancy. large-fetusedness - the average live weight of a nest and one piglet at birth. at 21 days and at weaning of a sow. the number of stillborns in the litter. the safety of piglets during the suckling period and at weaning. etc. [13]. As a rule. multiple pregnancies and large-fetusedness are negatively related. In many countries with developed pig breeding. active work is being carried out to increase the number of fetuses and preserve large-fetusedness. Under these conditions. among the classical methods of genetics. genomic analysis of multiple pregnancies is very promising. [17. 18. 7. 16].

One of the reasons for the low number of pig litter is intrauterine loss and stillbirth. The reasons for embryonic losses are diverse. based on genetic. functional. alimentary. less often mechanical. infectious and invasive factors.

According to many authors. embryonic mortality in pigs is $20-50 \%$ (Studentsov A.P.. 1934; Ladan P.E.. 1934; Betse I.. 1969; Prokoptsev V.M.. 1981; Levin K.L.. 1986). N.A. Martynenko (1971) explains the existence of this biological phenomenon with the inferiority of the gametes. the unpreparedness of the uterus for perception or its inability to ensure full bearing of the embryos and exogenous influences on the maternal organism. The morphophysiological features of the reproductive organs of pigs determine the possibility of maturation in the sexual cycle up to 30 and ovulation of 16-20 follicles (Voloschik P.D.. Pushkarsky V.G.. 1982). According to I.I.Sokolovskaya (1962). about 30-50\% of the eggs die before and after fertilization and at various stages of fetal development. In practice. the biological fertility of pigs is hardly used by $60-76 \%$ (Bazhin M.M.. 1954; Kvasnitsky A.V.. 1983; Milovanov V.K.. Sokolovskaya I.I.. 1964; Malinkin N.P.. 1994).

The purpose of this study was to study embryonic mortality. the number of normal. hypotrophic and stillborn piglets in pigs in the conditions of the complex.

\section{Materials and research methods}

The studies were carried out in the autumn-winter period in the conditions of the Makkaveevsky industrial complex of the Zabaikalsky Territory. The influence of the number of piglets in the nest on the quality of litter was analyzed on pigs of large white breed of 2-4 years of age. After farrowing. the number of piglets born in the nest was taken into account. including norm-weight (full-fledged. normotrophic). hypotrophic (smallweight) and stillborn (dead). The dependence of the quality of litter on the number of piglets in the litter was traced. Embryonic mortality was determined by the difference between the number of pregnancy corpus luteum on the ovaries (potential) and the number of live piglets in a litter (actual fertility). Intrauterine losses were recorded at the dated dates of gestation - 30. 45. 60. 75. 90. 105 days. The measurements of the linear dimensions of the fetus' body were carried out. Biometric processing of numerical material was carried out according to R.Z. Siraziev. and others [10]. 


\section{Results obtained and their discussion}

It is known that with timely insemination. the fertilization rate of ovulated ovules reaches 95-99.4\%. After fertilization. at different stages of prenatal development. up to $30 \%$ of embryos. offspring. fetuses and $10 \%$ die during birth.

The data we obtained on the dynamics of the linear dimensions of the fetal body and embryonic mortality are summarized in Table 1. According to statistical indicators. the death of pig embryos is highest before the formation of the placental connection. that is. during the period of histotrophic nutrition. By the 30th day of gestation. with a body length of $2.4 \square 0.03 \mathrm{~cm}$. intrauterine losses are $21.6 \square 7.64 \%$. The death of fetuses in subsequent periods of prenatal development is less intense. At 45 days of gestation at $6.2 \square 0.21 \mathrm{~cm}$ of fetal body length. embryonic mortality is $27.2 \square 7.03 \%$. at 60 days it increases only by $2.0 \%$. at 75 - by $8.4 \%$. at 90 - by $10.0 \%$ and in 105 days - by $11.2 \%$. That is. from 30 days to 105 days of gestation. intrauterine losses increase only 1.8 times. for the entire period of gestation this figure is 38.4 percent.

Table 1. Body length and embryonic mortality in pigs.

\begin{tabular}{|c|c|c|c|c|c|c|}
\hline \multirow{2}{*}{$\begin{array}{c}\text { Days of } \\
\text { pregnancy }\end{array}$} & $\mathrm{n}$ & \multicolumn{2}{|c|}{ Fetus body length $(\mathrm{cm})$} & $\begin{array}{c}\text { Growth } \\
\text { rate }\end{array}$ & $\begin{array}{c}\text { Embryonic mortality } \\
\mathrm{P} \% \pm \mathrm{m}_{\mathrm{P} \%}\end{array}$ \\
\cline { 2 - 5 } & & \multicolumn{2}{|c|}{ Lim } & $\mathrm{M} \pm \mathrm{m}$ & & \\
\cline { 2 - 5 } & $\min$ & $\max$ & & & \\
\hline 30 & 29 & 2.1 & 2.6 & $2.4 \pm 0.03$ & - & $21.6 \pm 7.64$ \\
\hline 45 & 40 & 5.7 & 7.0 & $6.2 \pm 0.21$ & 2.6 & $27.2 \pm 7.03$ \\
\hline 60 & 34 & 11.0 & 12.5 & $11.7 \pm 0.24$ & 1.9 & $29.2 \pm 7.79$ \\
\hline 75 & 27 & 13.0 & 15.3 & $14.3 \pm 0.41$ & 1.2 & $35.6 \pm 9.21$ \\
\hline 90 & 15 & 15.0 & 18.2 & $17.3 \pm 0.86$ & 1.3 & $37.2 \pm 12.47$ \\
\hline 105 & 38 & 17.2 & 24.2 & $21.6 \pm 1.27$ & 1.2 & $38.4 \pm 7.88$ \\
\hline
\end{tabular}

Scientists disagree on the effect of the number of embryos in the uterine horns on their survival. AV Kvasnitsky (1983) believes that the number of embryos in the uterine cavity does not lead to embryonic mortality and does not even reduce the intensity of growth and development. V.E. Shavkun and others (1986) note an inverse relationship between the survival rate of embryos and their number.

The fetus can die at any stage of embryogenesis. however. the greatest losses are observed in the so-called critical periods. in which its sensitivity to damaging influences is especially high. There are reports that critical phases fall on those periods when there is a change in the connection and metabolism of the embryo with the mother's body. According to P.G. Svetlov (1960). they coincide with the formation of close ties between the fetus and the mother's body (implantation and placentation).

According to modern concepts. the critical phases in the prenatal period of ontogenesis are the beginning of zygote cleavage. rupture of the transparent membrane. the time of embryo implantation and placentation. W.F. Pope. N.L. First (1985) in the first forty days of gestation established the death of $35-45 \%$ of embryos. Approximately $65 \%$ of all embryonic losses of pigs occur during the period from fertilization to implantation. $75 \%$ from fertilization to 25 days of age of embryos. and only $25 \%$ may die during the rest of the gestation period (Prokoptsev V.M.. 1981).

In a comparative analysis. we found that in a number of sows at different periods of pregnancy. the amount of corpus luteum on one of the ovaries was 1-5 less than the number of fetuses of the corresponding fertile horn. The discovered phenomenon of pig reproduction biology. in our opinion. indicates the migration of embryos into the contralateral horn in the early stages of gestation. Perhaps this phenomenon is associated 
with the search for the most favorable sites for implantation.

According to P. Dzink (1985). the migration of embryos into the body of the uterus in pigs begins on the sixth day after ovulation. and on the ninth day. some embryos pass into the opposite horn. The movement of embryos continues until day 12. According to the author. the uniformity of the placement of the embryos influences the course of pregnancy. According to D.I. Grudeva (1976). the period from 9 to 14 days. when the embryos are implanted. is considered especially difficult. During these two weeks. over $20 \%$ of embryos die. B.P. Khvatov (1954). I.I. Yanovsky (1969) believe that there are certain zones in the endometrium with optimal conditions for implantation - the largest number of blood vessels and nerve endings.

At the same time B.S. Volzhenin (1949). referring to the problem of multiple pregnancy in pigs. noted that any part of the uterine mucosa contains a sufficient supply of potential energy for the development of the fetus and can serve as a place for the formation of a fullfledged placenta.

According to our data. the formation of a placental connection in large white pigs occurs at 30 days of gestation [9. 11]. by which time the embryonic mortality rate in sows is $21.6 \%$. As the pregnancy progresses. the difference in the size of the fetuses increases markedly. If during a 30-day pregnancy the difference between the maximum and minimum values of the body length of the pre-fetus was $0.3 \mathrm{~cm}$. then at 45 days in fetuses it increases to $2.3 \mathrm{~cm}$. and at 105 days it already reaches $7.0 \mathrm{~cm}$. The growth rate of fetuses is highest at 45 days of gestation (2.6). less at 60 days of gestation (1.9). then it decreases slightly and remains at this level until the end of pregnancy. Thus. embryos grow faster in the first half of embryogenesis.

Practically in the litter of pigs. fully developed. hypotrophic and dead piglets are found. Piglets born with a live weight of more than $1.0 \mathrm{~kg}$ subsequently grow healthy. develop normally and have a high safety. We also traced the dependence of the quality of litter on the number of piglets in the nest in sows in conventionally separated groups (Table 2).

Table 2. Dependence of the usefulness of piglets on the size of the litter.

\begin{tabular}{|c|c|c|c|c|c|}
\hline \multirow[b]{2}{*}{$\mathrm{n}$} & \multirow{2}{*}{$\begin{array}{l}\text { The number of } \\
\text { piglets in the litter } \\
\text { (conditional group }\end{array}$} & \multirow{2}{*}{$\begin{array}{c}\text { Total } \\
\text { piglets } \\
\mathrm{n}\end{array}$} & \multicolumn{3}{|c|}{ Of them } \\
\hline & & & $\begin{array}{c}\text { Full-fledged } \\
\mathrm{P} \% \pm \mathrm{m}_{\mathrm{P} \%}\end{array}$ & $\begin{array}{c}\text { Hypotrophic } \\
\mathrm{P} \% \pm \mathrm{m}_{\mathrm{P} \%}\end{array}$ & $\begin{array}{l}\text { Stillborn } \\
\mathrm{P} \% \pm \mathrm{m}_{\mathrm{P} \%}\end{array}$ \\
\hline 1 & $2-6$ & 82 & $96.3 \pm 2.08$ & $3.7 \pm 2.08$ & - \\
\hline 2 & $7-11$ & 819 & $80.8 \pm 1.38 * * *$ & $13.3 \pm 1.17 * * *$ & $5.9 \pm 0.82 * * *$ \\
\hline 3 & $12-16$ & 666 & $73.9 \pm 1.70 * * *$ & $18.0 \pm 1.39 * * *$ & $8.1 \pm 1.05^{* * *}$ \\
\hline 4 & $17-21$ & 41 & $53.7 \pm 7.79 * * *$ & $31.7 \pm 7.27 * * *$ & $14.6 \pm 5.50^{* * *}$ \\
\hline \multicolumn{3}{|c|}{ Correlation coefficient } & $\mathrm{r}=-0.98$ & $\mathrm{r}=+0.98$ & $\mathrm{r}=+0.98$ \\
\hline
\end{tabular}

In a litter where 2-6 piglets were born. full-fledged ones make up 96.3 $\square 2.08 \%$. 7-11 $80.8 \square 1.38 \%$. $12-16-73.9 \square 1.70 \%$. $17-21-53.7 \square 7.79 \%$. That is. there is an inverse relationship between fertility and usefulness of piglets $(r=-0.98)$. The difference between the first and subsequent groups is significant for the third threshold [8].

These strong litters will have their own stunted piglets. which will not get mother's milk due to the fact that there is simply not enough nipples for everyone. because even now it is not uncommon for litters of 18 pigs. and the working nipples in the sows are no more than 14. In Russia in such cases coped easily. seating piglets from multiple sows to infertile. And if there are more multiple births? Here. one way is to artificially raise piglets left without mother's milk or weak. Various milk substitutes are usually used for them. but the rest of 
their living conditions remain the same and they are usually slaughtered by their stronger brethren. They get less food. heat and light. and in such conditions they grow poorly and often die.

In Russia. piglets weighing less than $1 \mathrm{~kg}$ were considered unviable. therefore pigbreeders in many farms immediately killed small pigs so as not to bother caring for them. in other farms they waited 2-3 days when the weak and low-weight themselves would die and then officially survived came like newborns. This practice continues to this day in many farms.With this approach. the pig-breeders protected themselves from the decrease in earnings associated with a high mortality. and the multiple births of 9-10 piglets per farrow. instead of the actual 12 . were associated with the reproductive qualities of the sow. The mortality rate was recorded at $3-5 \%$. instead of the actual $20 \%$. Thus. no more than 8 piglets reached the end of fattening. and if we take into account the imperfect work on reproduction with the broodstock. an average of 16 piglets were received from one sow at 2 farrowings per year [12].

We found a direct relationship between the number of piglets in the litter and the number of hypotrophic pigs in it $(\mathrm{r}=+0.98)$. that is. the more born. the more weak (Fig. 1). With low live weight. $3.7-2.08 \%$ were found; $13.3 \square 1.17 \%$; $18.0 \square 1.39 \%$; $31.7-7.27 \%$. respectively. in the above conditional groups.
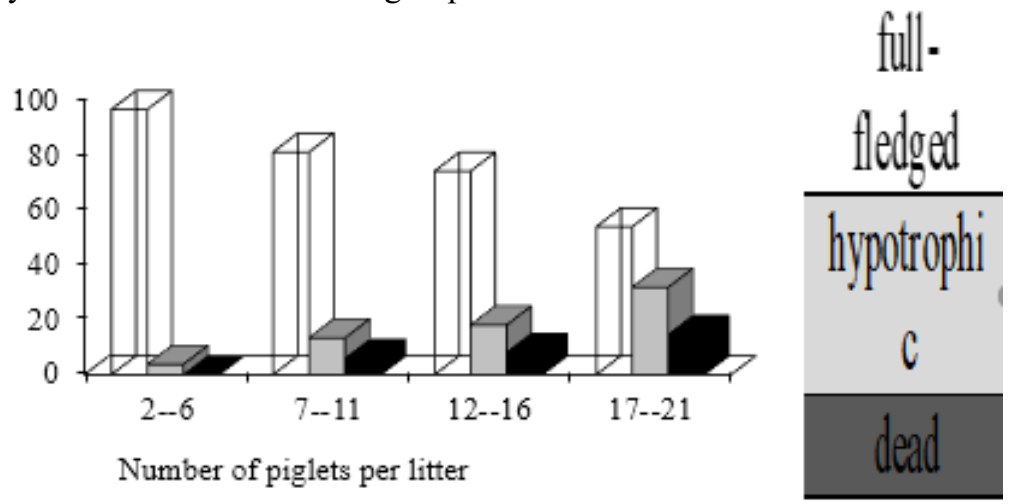

Fig.1. The effect of multiple births on the usefulness of piglets.

The reasons for the birth of hypotrophic piglets are subdivided into endogenous - $47 \%$. exogenous $-47 \%$ and genetic - $6 \%$. In the South Urals region. as confirmed by the experiments of A.I. Kuznetsov [3]. the dependence of the growth in the number of births of weak piglets on the season of the year was established from 20 to $45 \%$. In the Belgorod region at pig farms. according to E.A. Shipilov [15]. 20-25\% of weak young were born. which did not grow well. At the same time. the safety during the period of further rearing was extremely low - from 12 to $53 \%$.

Smaller piglets do not compete for life in nests with larger littermates. and $60-80 \%$ of them die in the first days of life. and the surviving piglets are far behind in growth. that is why they are subject to culling [2. 6]. With intensive technologies of pig breeding. $11-45 \%$ of hypotrophic piglets are born. Moreover. the longer the complex works. the greater the yield of young animals with a low live weight at birth [5]. Therefore. improving the safety and rearing of low-birth-weight piglets remains the most important problem of industrial pig breeding. For a long time. on domestic pig farms. preventive clinics (pigbalii). nest dens were arranged for piglets. and houses with heating were also made for them. But modern life forces us to improve what was used yesterday.

In Europe. this problem has always been very acute. they are not used to scattering newborn pigs. Farmers make every effort to keep as many piglets as possible and generate 
additional income as a result. One of the leading developments of Dutch pig breeders is the creation of special incubator houses. the so-called "kuvez" for weak piglets. Kuvez (French couveus. "incubator") is a device with automatic feeding and maintaining the optimum temperature. in which a premature or sick newborn is placed. The novelty was appreciated at its true worth in European countries. and the first buyers appeared in Russia.

Kuvez is a one-piece plastic chamber with dimensions of $150 \times 100 \times 60$. The front wall is a transparent door with a slot in the top parts for fresh air access. Drafts in the chamber are initially impossible. Special germicidal lamps have several incandescent levels and you can easily change the temperature. Floors are slatted. and waste products fall into the pallet. from where they are regularly washed into the general drain of the pigsty. Each kuvez is equipped with a feed trough for combined feed and drinkers for water and milk replacer. Water and milk are continuously supplied to the drinking bowls by a pump that can serve up to 20 piglets. Usually they are mounted above the machines where suckling sows are kept. and the operator can easily observe what is happening with his wards. The only stress on the operator is adding fresh feed to the piglets' feeder. The incubation period lasts 20-25 days.

Weak piglets are placed in incubators not immediately after birth. but on the 3rd-4th days. Why is this done? Until the sow runs out of colostrum. each pig in the first hours of life should receive it. thereby strengthening its immunity. Clostral immunity ensures the same survival of piglets. surrounded by a mass of pathogenic bacteria. In order for the weak piglets to get colostrum. their stronger counterparts are temporarily deposited several times a day in a special box. and the weak ones are placed in the mother's milkiest nipples. After sows' colostrum is exchanged for milk. the weakest piglets from several litters are collected and placed for artificial rearing. Usually 10-12 piglets are housed in a kuvez. where they develop in a confined space. Of course. even in comfortable conditions. losses occur and not everyone can survive until the end of the period. but the safety is still great. According to European statistics. earlier. only $30 \%$ of weak piglets survived under the sow. after using incubators. already $70 \%$. In addition. if a piglet raised under artificial conditions weighed on average $0.5 \mathrm{~kg}$ less than its counterparts raised by its mother. then it quickly caught up and surpassed them in terms of growth rates. This was due to the fact that artificial piglets were not subjected to additional stress from parting with their mother. while suckling pigs experienced severe stress and lost their appetite for several days. Each farrowing room. after weaning of piglets and complete emptying. is cleaned and disinfected. the incubators are also subjected to these measures and it is quite easy to do this. because they are removable and do not have any gaps where any dirt can collect. The pump and supply pipes are also easily flushed out [12].

The sore question of our pig breeders is where to get the funds to purchase these miraculous kuvezes? It must be said right away that this whole system "chamber-pumppipes-drinkers" is not very cheap.

It is hoped that the project on the introduction of the technology of preservation of young animals. using a fully equipped domestic incubator. in Russia will be placed in the highest priority areas of development of animal husbandry. If we have learned to increase the multiple pregnancy of sows. then it is simply mediocre to lose additional piglets and our own profit.

According to our observations. the number of dead pigs also increases $(r=+0.98)$ as the number of newborns in the litter increases (Fig. 1). In the group with 2-6 piglets there were no dead. with 7-11 out of 819 newborns the dead were $5.9 \square 0.28 \%$. with $12-16$ out of 666 piglets there were $8.1 \square 1.05 \%$. 17-21 - 14.6 $\square 5.50$ percent. Our results differ from the data of A.I. Varganov (1971). according to which in the litters of Urzhum breed pigs. where 1517 piglets were born. the dead were $46.7 \%$. with $18-19-57.1 \%$. 
According to K. Bratanov et al. (1984). with traditional technology. the number of stillbirths is $5-8 \%$. with an industrial content. it can exceed 15-20 percent. According to G. Randall. R. Renny (1970). stillbirth is more often observed in multiple litters. as well as in sows with a shortened fertile period. G. Fisher (1975) notes an increase in the proportion of stillbirths with an increase in multiple births of more than 12 piglets.

Even F.F. Müller (1955). for the first time. developing methods for the prevention and treatment of malnutrition of young pigs. found that under the conditions of small pig farms under good feeding and maintenance conditions. malnutrition of pigs was noted in $10-12 \%$ of cases. In the conditions of intensive production technologies. there was a decrease in fertility. multiple fertility of sows. an increase in the number of small piglets up to $16-45 \%$. safety was $12-53 \%$. stillborns - up to $15-20 \%$ [5. 6].

\section{Conclusion}

It should be said that many reasons leading to intrauterine death and stillbirth allow embryonic mortality to be considered a factor of natural selection at the earliest stages of embryonic development. During embryogenesis. embryos with reduced constitutional strength die. In our opinion. it is necessary to increase the fertility of pigs by preventing intrauterine death of piglets. especially in the early stages of embryogenesis. The main task of domestic pig breeders and scientists is considered to be the need to increase the multiple birth rate to 12-16 piglets per farrowing. it is necessary to conduct selection according to the number of nipples. early maturity. body length. preservation of large-fetusness using hightech methods of feeding. rearing. genetic potential of domestic and foreign breeds. modern methods of directional selection. artificial insemination. stimulation of the reproductive function of sows and modern biotechnological techniques.

\section{References}

1. N.S. Gegamyan. N.V. Ponomarev. A.L. Chernogorov An effective system of pork production (experience. problems and solutions). in 2 parts 2 nd ed.. add. and revised (2010)

2. A.I. Grishin. Reproductive function of sows when feeding them a suspension of chlorella: thesis, Cand. of Agr. Sciences, 132 (2013)

3. A.I. Kuznetsov Physiological immaturity of pigs: factors that determine its occurrence. features of the course and manifestation of the most important functions of the body. methods of prevention and correction: abstract thesis, 43(1996)

4. N.A. Loban. O.Ya. Vasilyuk. E.V. Pishchelka Results and prospects of breeding work with the Belarusian large white breed of pigs Actual problems of intensive development of animal husbandry, 20-1, 22 -31 (2017)

5. N.V. Ponomarev The main factors in the intensification of pork production at enterprises of various capacities: abstract thesis, Dr. of Agr. Sciences 36 (1997)

6. G.S. Pokhodnya. A.I. Grishin. R.A. Strelnikov. E.G. Fedorchuk. V.V. Shablovsky Increasing the productivity of broodstock pigs: monograph, 488 (2013)

7. V.V. Semenov. L.V. Kononov. V.I. Lozovoy The relationship of the ESR gene with the reproductive qualities of pigs Collection of Scientific, Works of the Stavropol Research Institute of Livestock and Forage Production 3, 7, 342-347 (2014) 
8. R. Z. Siraziev On the issue of embryonic mortality and stillbirth of piglets Veterinariya, 6, 36-38 (1999)

9. R. Z. Siraziev Morphofunctional changes in the uterus of pigs under various physiological conditions and in the experiment; thesis, 350 (1999)

10. R.Z. Siraziev. L.M. Malakshinova. N.B.Saduev. G.A. Igumnov. R.Ts. Tsydypov Statistical analysis of mathematical data in biology (for independent work) Approved by the Ministry of Agriculture of the Russian Federation as a textbook for students of higher educational institutions studying in the specialties 310700 - "Zootechnics" and 31080 - "Veterinary 73 p (2005)

11. R.Z. Siraziev Histological and histochemical characteristics of areoles in the placenta of pigs , 57, 5, 379-386 (2015)

12. A. V. Topchin The safety of young animals is the most important factor in increasing profitability in pig breeding, Bulletin of VNIIMZh,3 (7), 38-42 (2012)

13. A.M. Khokhlov. D.I. Baranovsky Reproductive qualities of sows depending on biological and technological factors, Bulletin of the Bryansk State. Agr. Academy, 3 (61), 37-41 (2017)

14. A. I. Shendakov Assessment of the potential of multiple births in modern breeding of breeding pigs, Bulletin of Agrarian Science, 2 (77), 77-84 (2019)

15. E. A. Shipilov Influence of live weight of piglets at birth on their growth. development and reproductive functions; thesis, 136 (2002)

16. S. Berg Imputation to whole-genome sequence using multiple pig populations and its use in genome-wide association studies Genetics. Selection. Evolution: GSE, 51 (1), 32 p (2019)

17. A. Huashui Genetic diversity. linkage disequilibrium and selection signatures in chinese and western pigs revealed by genome-wide SNP markers, 7, 22 (2013) //https://doi.org/10.1371/journal.pone.0056001

18. R. Wellmann Genomic selection using low density marker panels with application to a sire line in pigs, Genetics Selection Evolution, 45, 28 (2013) 